\title{
Endovascular repair of type $B$ aortic dissection with the restrictive bare stent technique: morphologic changes, technique details, and outcomes
}

This article was published in the following Dove Press journal:

Therapeutics and Clinical Risk Management

\author{
Binshan Zha ${ }^{1,2}$ \\ Geliang $X u^{\prime}$ \\ Huagang Zhu ${ }^{2}$ \\ Wentao $\mathrm{Xie}^{2}$ \\ Zhigong Zhang ${ }^{2}$ \\ Yongsheng $\mathrm{Li}^{2}$ \\ Peng Qiu ${ }^{3}$ \\ 'Department of General Surgery \\ and Anhui Province Key Laboratory \\ of Hepatopancreatobiliary Surgery, \\ Affiliated Provincial Hospital of Anhui \\ Medical University, Hefei, People's \\ Republic of China; ${ }^{2}$ Department \\ of Vascular and Thyroid Surgery, \\ Department of General Surgery, \\ The First Affiliated Hospital of Anhui \\ Medical University, Hefei, People's \\ Republic of China; ${ }^{3}$ Department of \\ Vascular Surgery, Fourth Affiliated \\ Hospital of Anhui Medical University, \\ Hefei, People's Republic of China
}

Purpose: The aim of this study was to present our experience and assess the morphologic changes of the descending aorta after the restrictive bare stent (RBS) technique in the treatment of type B aortic dissection (TBAD).

Patients and methods: A retrospective study was conducted of 22 consecutive patients with TBAD who underwent RBS treatment between February 2012 and June 2016. Indications for the RBS procedure included radiological evidence of true lumen (TL) compression or collapse and/or tortuosity index (TI) of the descending aorta $>1.4$. Technique success, descending aorta morphology, and clinical outcomes were evaluated.

Results: The technical success rate was $100 \%$. Patients treated with the RBS technique were often accompanied by TL collapse $(45.5 \%)$ or TI $>1.4(59.1 \%)$. One-month postoperative computed tomography angiography showed that the taper ratio, oversizing ratio of the stent graft, and TI values were significantly decreased compared with preoperative computed tomography angiography values $(P<0.05)$. The 30 -day mortality rate was $0 \%$. In total $95.2 \%$ had a thrombosed false lumen in the segment of aortic coverage, and TL diameters were increased in $40.3 \% \pm 11 \%$ (mean $\pm \mathrm{SD}$ ) and $37.5 \% \pm 17.9 \%$ of patients in the thoracic and abdominal segments, respectively. During the follow-up from 16 to 64 months ( $33 \pm 19$ months), no distal stent graft-induced new entry, endoleak, and paraplegia were observed. One patient died from rupture of a chronic TBAD with aneurysm degeneration.

Conclusion: Mid-term outcomes showed RBS to be a flexible and feasible approach to repair TBAD. RBS corrects the descending aorta morphology and promotes TL expansion in most patients, but the rupture of chronic TBAD with aneurysm degeneration was not prevented in all patients.

Keywords: bare metal stent, stent graft, anatomy, type B aortic dissection, thoracic endovascular repair, aneurysm

\section{Introduction}

Thoracic endovascular aortic repair (TEVAR) has become an accepted and preferred approach for type B aortic dissection (TBAD). ${ }^{1,2}$ However, mid-term and long-term follow-up studies have demonstrated prominent distal stent-related complications. ${ }^{3-5}$ Previous studies have shown that the incidence of distal stent graft (SG)-induced new entry (SINE) ranges from $1.08 \%$ to $34.78 \%$ and is associated with a mortality rate of $25 \% .^{6-11}$ In addition, $14 \%-26 \%$ of patients will develop distal thoracic aortic enlargement after TEVAR. ${ }^{12}$ Therefore, the prevention of distal stent-related complications is an important issue. 
The mechanisms of distal stent-related complications depend on several factors. First, mismatch between the comparatively larger size of the SG and the smaller size of the highly tapered true lumen (TL) of the dissected descending aorta is one of the main causes of complications, and excessive radial force from oversizing can result in damage to the fragile intima. Second, excessive spring-back strength from the tortuosity of the descending aorta fixation site makes the intima potentially vulnerable..$^{3,6-8,13,14}$ Third, the perfusion of the false lumen (FL) will lead to long-term complications in patients, such as aneurysmal degeneration and rupture. ${ }^{15}$

The provisional extension to induce complete attachment (PETTICOAT) technique was first reported to induce positive aortic remodeling. ${ }^{16}$ But it was a concern that distal SINE could occur prior to the placement of the distal bare stent. Therefore, the restrictive bare stent (RBS) technique has been proposed to prevent distal SINE and improve aortic remodeling by reducing the taper ratio (TR) and the distal oversizing ratio (OR). ${ }^{17-20}$ The key point of this technique is to preplace a bare stent in the descending aorta at the level of the intended distal end of the SG. However, the tortuous descending aorta and chronic TBAD with aneurysm degeneration are not involved in these studies. Herein, we retrospectively review our experience and assess the morphologic changes and the clinical outcomes of the RBS technique for TBAD with TL compression or collapse and/or tortuosity index $(\mathrm{TI})>1.4$.

\section{Materials and methods}

\section{Study design and patient population}

The Institutional Review Board of First Affiliated Hospital of Anhui Medical University approved this study. Signed informed consent was obtained from each patient involved in the study. Patients treated with the RBS procedure between February 2012 and June 2016 at our institution were included. The definitions of TBAD stage used in this study are as follows: acute phase ( $\leq 14$ days from onset to TEVAR), subacute phase ( $>14$ days and $<3$ months), and chronic phase ( $>3$ months). Chronic TBAD with aneurysm degeneration was defined as aortic dissection progression to a thoracic aneurysm with a maximum descending aorta diameter $\geq 55 \mathrm{~mm} .^{21}$ Indications for the RBS procedure included radiological evidence of TL compression or collapse and/or TI $>1.4$ (Figure 1A). Connective tissue disease, intramural hematoma, and penetrating atherosclerotic ulcer were excluded from the study to meet the RBS inclusion criteria. The criteria for complicated TBAD included distal malperfusion, impending rupture, resistant hypertension, and persistent chest or back pain. Patients with TBAD who met one or more of the criteria were considered for emergency endovascular repair.

\section{Endovascular procedure}

All procedures were performed under general anesthesia. Intraoperative thoracic and abdominal aortic angiography was routinely performed to assess the patency of the visceral vessels and their origin from the TL or the FL (Figure 1B and $\mathrm{C}$ ). The size of the proximal SG was the diameter of the proximal nondissected aorta, usually at the ostia of the left subclavian artery. Some patients required partial or total exclusion of the left subclavian artery to obtain an adequate proximal landing zone if the right vertebral artery was patent and the left one was not dominant. Technical success was defined as SG deployment without type I endoleak, open surgical conversion, or death within $24 \mathrm{~h}$ of the operation.
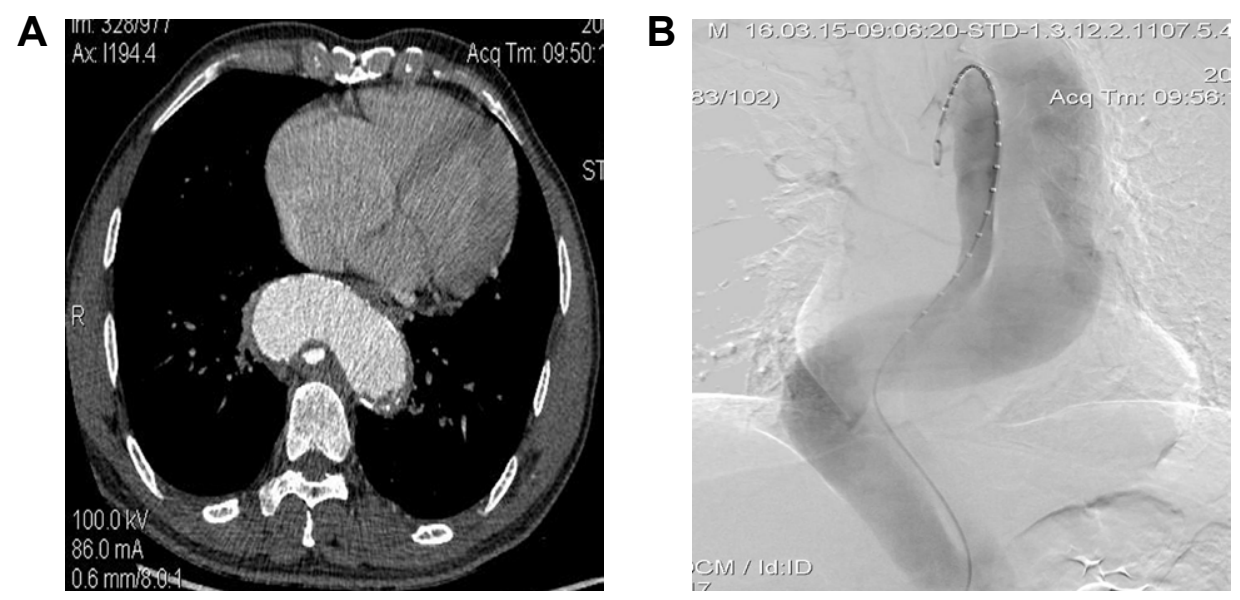

Figure I (Continued) 


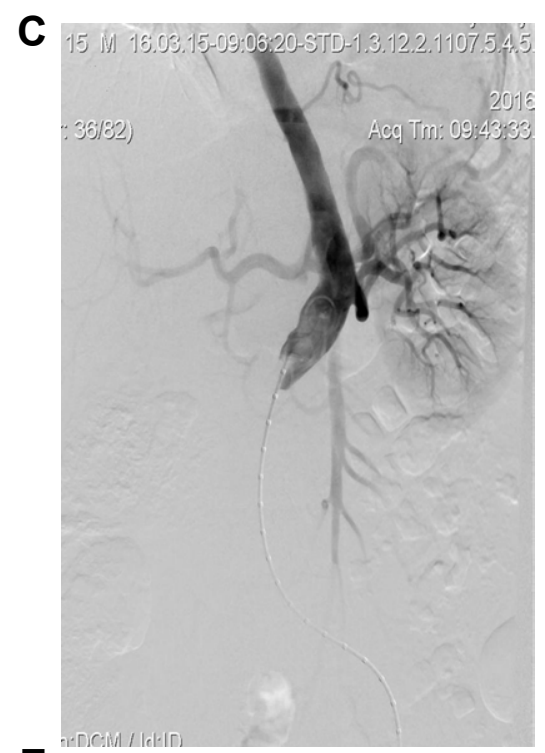

E

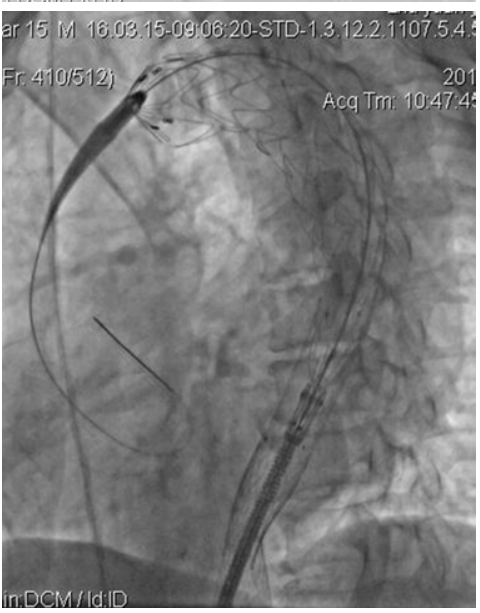

G

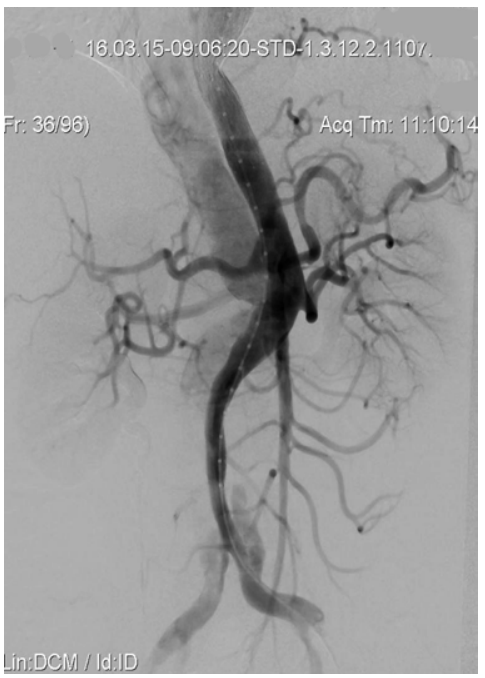

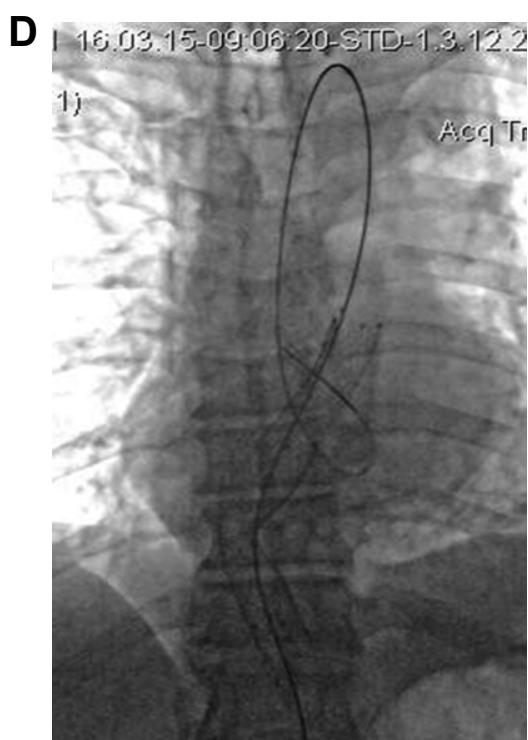

$\mathbf{F}$

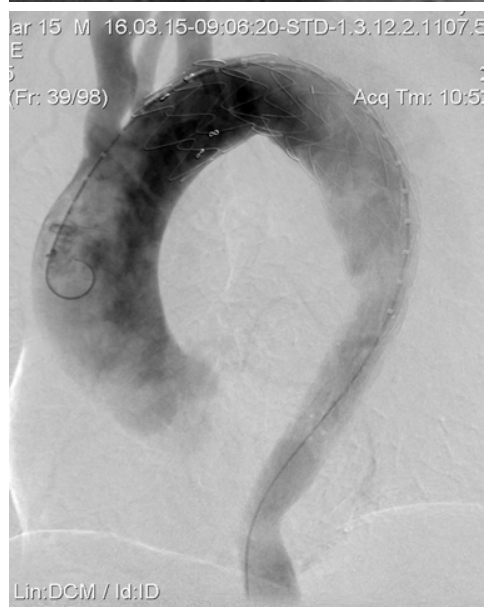

H

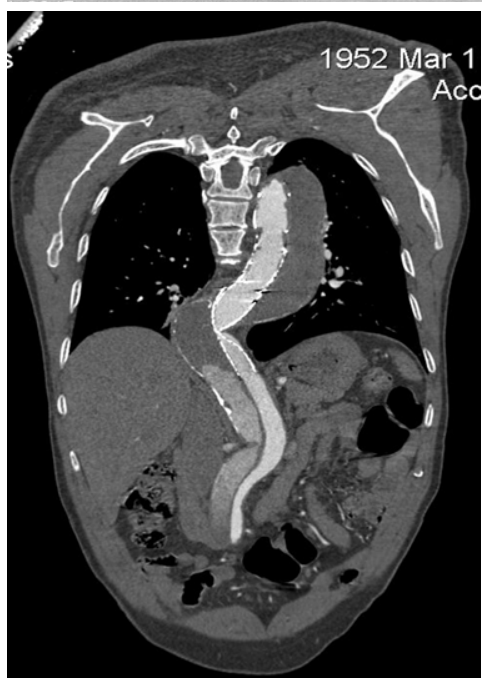

Figure I A 63-year-old man who presented with 3 months of chest and back pain was diagnosed with chronic TBAD with aneurysm degeneration (using patient 4 as an example). Total stent graft diameter and length were $36 \mathrm{~mm}$ and $200 \mathrm{~mm}$, respectively. Two RBSs were implanted, and the diameter of the proximal RBS was $26 \mathrm{~mm}$.

Notes: $(\mathbf{A}-\mathbf{C})$ Preoperative CTA and intraoperative angiography showing severe tortuosity of the descending aorta $(\mathrm{TI}=\mathrm{I.62})$ and collapsed $\mathrm{TL}(\mathrm{TR}=0.89, \mathrm{OR}=9.9)$. Intraoperative angiography verifying the TL collapse. (D, E) Two RBSs (black arrow) were first placed to reduce the angle between the distal end of the stent and the aorta and to locate the stent fixation site in a straight portion of the descending aorta. The stent graft was then deployed to seal the proximal entry tear, leaving its distal end partially within the RBS. (F, G) Postoperative angiography showing satisfactory repair of the dissected aorta using the RBS procedure. Meanwhile, the tortuosity of the descending aorta was corrected. TL was obviously expanded, and no endoleak was observed. (H) CTA at postoperative year I showing satisfactory occlusion of the aortic dissection with re-expansion of the TL and RBS collapse.

Abbreviations: TR, taper ratio; OR, oversizing ratio of the stent graft; TI, tortuosity index; TBAD, type B aortic dissection; RBS, restrictive bare stent; TL, true lumen; CTA, computed tomography angiography. 
The RBS technique was previously reported in detail and is based on the following strategy:

1. The diameter of the RBS was selected according to the greatest diameter of the TL at the intended distal edge of the RBS. No oversize was adopted for the selection of the bare stent.

2. The RBS was first placed into the compressed or collapsed TL to restrict the spring-back force of the distal SG edge while expanding the TL. If a tortuous descending aorta and TL collapse existed simultaneously, the RBS helped pave the road for advancing of the delivery system and reduced the angle between the distal end of the stent and the aorta.

3. More than one RBS was placed to maintain the stability and locate the descending aorta fixation site in a relatively straight portion (Figure 1D). The length and numbers of the RBS were determined by tortuosity location and TL compression degree. Considering iatrogenic malperfusion, RBS placement over visceral artery ostia was avoided.

4. The Sinus-XL stent (Optimed, Ettlingen, Germany) was used as the RBS in all patients. The RBS was positioned proximally to anticipate $30-40 \mathrm{~mm}$ of overlap with the distal end of the proximal SG.

5. After the RBS was deployed and secure, the SG, approximately $10 \%-15 \%$ oversized, was introduced into the proximal landing zone and deployed to seal the proximal entry tear, leaving its distal end partially within the RBS (Figure 1E). No postdeployment ballooning was used. Completion aortography was then performed to ensure the coverage of the proximal entry tear and to visualize the side branches (Figure 1F and $\mathrm{G}$ ).

6. In cases with persistent malperfusion syndrome, an adjunctive procedure (distal bare stent extension or open surgery) was performed.

\section{Follow-up}

Computed tomography angiography (CTA) was performed before discharge and at 1, 3, 6, and 12 months postoperatively, followed by annual examinations in the extended follow-up period (Figure 1H).

\section{Data collection and definitions}

CTA images were collected before and after the procedure. The definitions and mathematical measurements of descending aorta morphology are as follows (Figure 2):

1. Prestent and poststent TR: the proximal vs the distal landing area, defined as $1-\left(\mathrm{X}_{\mathrm{A}} / \mathrm{X}_{\mathrm{PRO}}\right)$. Prestent and poststent OR: the oversizing ratio at the distal end of the $\mathrm{SG}$, defined as $\left(\mathrm{X}_{\mathrm{SG}} / \mathrm{X}_{\mathrm{A}}\right)-1 . \mathrm{X}_{\mathrm{A}}$ is the area of the TL at the presumed distal end of the $\mathrm{SG} ; \mathrm{X}_{\mathrm{PRO}}$ is the presurgery area of the proximal landing zone; $\mathrm{X}_{\mathrm{SG}}$ is the distal size of the fully expanded stent before insertion. After stenting, $\mathrm{X}_{\mathrm{A}}$ is the area of the $\mathrm{TL}$ at the real distal end of the $\mathrm{SG}$, and $\mathrm{X}_{\mathrm{PRO}}$ is the poststent area of the proximal landing zone. $\mathrm{X}_{\mathrm{SG}}$ is the distal area of the partially expanded stent after insertion. ${ }^{7}$

2. TI was defined as the intra-aorta midline length divided by the linear distance between the left subclavian artery and the celiac trunk. ${ }^{22}$

3. TL expansion was assessed separately at the level of the descending thoracic aorta and the abdominal aorta (divided at the level of the celiac trunk). The aortic TL diameter and the total aortic diameter at the site of maximal aortic disease were measured using sectional computed tomography scans. The postoperative diameters were measured at similar locations and were subsequently compared with preoperative computed tomography scan data.

4. The status of the TL was qualitatively assessed as patent, partially thrombosed, or completely thrombosed in the
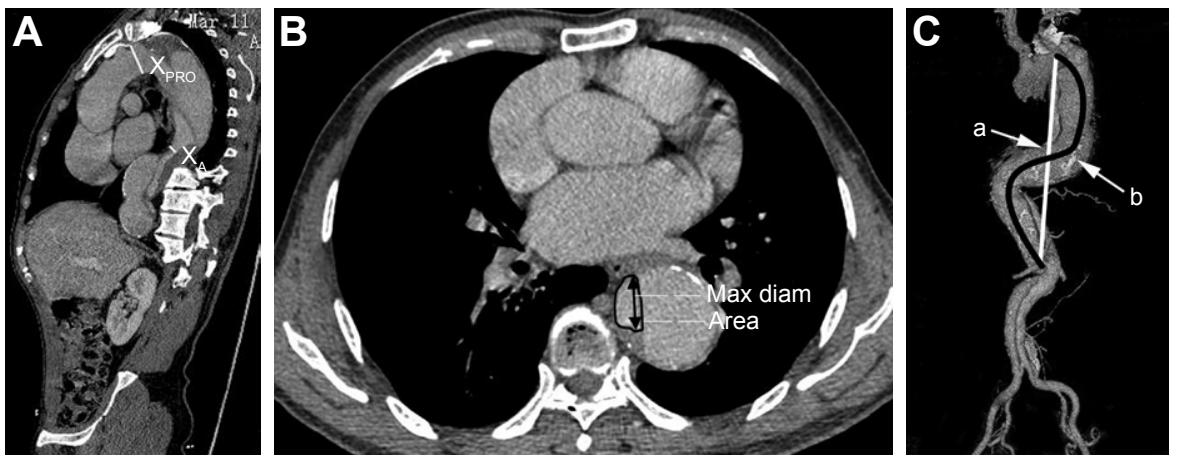

Figure 2 Measurement of the local and global anatomy of the descending aorta (TR, OR and TI).

Notes: $(A)$ Sagittal computed tomography view before TEVAR showing the size of the presumed proximal landing zone $\left(\mathrm{X}_{\mathrm{PRO}}\right)$ and the presumed distal landing zone $\left(\mathrm{X}_{\mathrm{A}}\right)$. (B) Maximal diameter and area of $\mathrm{X}_{\mathrm{A}}$. $(\mathbf{C}) \mathrm{TI}=\mathrm{b} / \mathrm{a}$, defined as the length of the midline (b) within the aorta divided by the linear distance (a) between the left subclavian artery and the celiac trunk.

Abbreviations: TR, taper ratio; OR, oversizing ratio of the stent graft; TI, tortuosity index; TEVAR, thoracic endovascular aortic repair; Max, maximal; diam, diameter. 
segments of aortic coverage, the distal SG, and the abdomen, respectively.

\section{Statistical analyses}

Patient demographic characteristics are summarized as the mean \pm SD for continuous data and as a number (\%) for categorical data. Differences in descending aorta morphology parameters were compared using a two-sample $t$-test for normally distributed data or the Mann-Whitney $U$-test for data with nonnormal distributions. All statistical assessments were two-tailed and were considered significantly different at $P<0.05$. Statistical analyses were conducted with SPSS Statistics 22 software (IBM Corporation, Armonk, NY, USA).

\section{Results}

\section{Patient characteristics}

A total of 22 patients (17 men; median age 54.9 years, range 33-75 years) underwent the RBS procedure. Of these patients, 8 were treated during the acute phase, 5 were treated during the subacute phase, and 9 were treated during the chronic phase. A total of $66.7 \%$ (6/9) of the chronic phase patients exhibited chronic TBAD with aneurysm degeneration. Five patients required emergency surgery due to distal malperfusion $(n=4)$ and dissection impending rupture $(n=1)$. The demographic and comorbidity data are summarized in Tables 1 and 2 .

A subset of patients treated with the RBS technique exhibited TL collapse $(10 / 22,45.5 \%)$ and TI $>1.4$ (13/22, $59.1 \%$ ). The preprocedural CTA revealed TR, OR, and TI values of $0.79 \pm 0.08,4.74 \pm 2.6$, and $1.44 \pm 0.17$, respectively (Tables 1 and 2).

\section{Procedure information}

The technical success rate was 100\%. Four SG systems were used in this study: Zenith TX2 (Cook Medical, Bjaeverkov, Denmark; 3/22, 13.6\%), Ancura (Lifetech, Shenzhen, People's Republic of China; 4/22, 18.2\%), Grikin (Grikin, Beijing, People's Republic of China; 1/22, 4.5\%), and Captivia (Medtronic, Santa Rosa, CA, USA; 14/22,

Table I Patient characteristics

\begin{tabular}{l|l|l|l}
\hline Variable & $\begin{array}{l}\text { Preoperation } \\
\text { CTA }\end{array}$ & $\begin{array}{l}\text { Postoperation } \\
\text { CTA }\end{array}$ & P-value \\
\hline TR & $0.79 \pm 0.08$ & $0.50 \pm 0.15$ & $<0.001$ \\
OR & $4.74 \pm 2.68$ & $1.93 \pm 1.03$ & $<0.001$ \\
TI & $1.44 \pm 0.17$ & $1.32 \pm 0.16$ & $<0.05$ \\
\hline
\end{tabular}

Note: Continuous data are presented as mean \pm SD.

Abbreviations: CTA, computed tomography angiography; OR, oversizing ratio of the stent graft; TI, tortuosity index; TR, taper ratio.
63.7\%). In total, 35 RBSs were placed on the distal side. All of the descending aorta fixation sites were located in the straight aortic portion. Three patients who presented with static obstruction were treated with additional procedures after the RBS procedure. Detailed procedural information is presented in Tables 2 and 3.

\section{Descending aorta morphologic changes and remodeling}

The 1-month postoperative CTA scans showed that the TR and OR values decreased significantly after the RBS technique $(0.76 \pm 0.08$ vs $0.50 \pm 0.15, P<0.001$ and $4.74 \pm 2.68$ vs $1.93 \pm 1.03, P<0.001$, respectively). Furthermore, the TI values were also notably lower than those before surgery (1.44 \pm 0.17 vs $1.32 \pm 0.16, P=0.029)$. Additionally, the percentages of completely thrombosed FL in the segments of aortic coverage, the distal SG, and the abdomen were $95.2 \%(21 / 22)$, 63.6\% (14/22), and 13.6\% (3/22), respectively. Moreover, the TL diameter expanded significantly in the thoracic and abdominal segments compared with the preoperative TL diameter $(40.3 \% \pm 11 \%$ and $37.5 \% \pm 17.9 \%$, respectively).

\section{Thirty-day mortality and aortic-related morbidity}

The 30 -day mortality rate was $0 \%$. The morbidity at 30 days included RBS collapses (13.6\%); no instances of endoleak, stroke, paraplegia, retrograde dissection, or visceral artery ischemia were observed.

\section{Post-30-day aortic-related morbidity and mortality}

We followed up all patients from 16 to 64 months (33 \pm 19 months). No distal SINE, endoleak, stroke, paraplegia, or reintervention events occurred. The $>30$-day morbidity included RBS collapse (13.6\%, within 30 days) in patients 2, 3, and 4 (Table 4) (Figure 1H). The 1-month follow-up CTA revealed that no collapsed stents re-expanded. Despite this unsatisfactory morphologic result, the patients did not complain of clinical symptoms.

The $>30$-day aortic-related mortality rate was $4.5 \%$. One patient (patient 3 ) presented with chronic TBAD with aneurysm degeneration (total aortic diameter $=71 \mathrm{~mm}$ ) and underwent the procedure. Intraoperative digital subtraction angiography showed that the primary tear flap was successfully excluded with no endoleak, and the distal reflux was limited to the abdominal segment. Follow-up CTA at 1 year showed an increased FL diameter, and the total aorta 


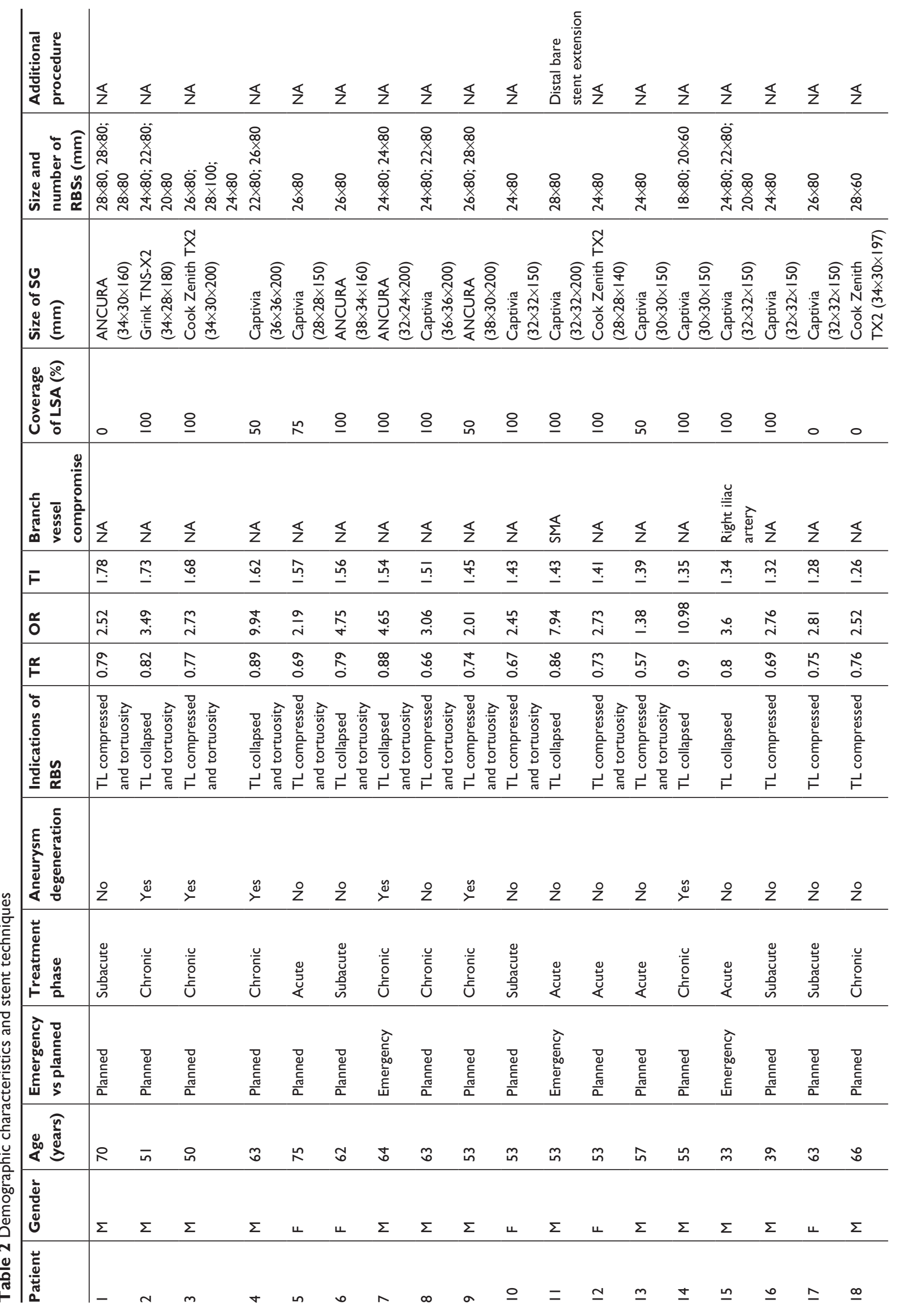



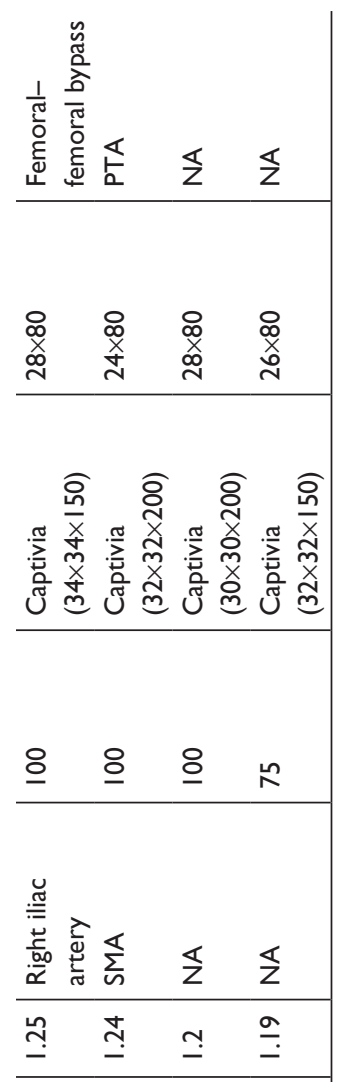

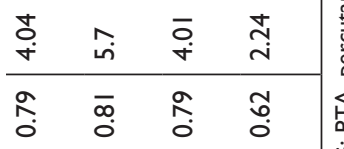
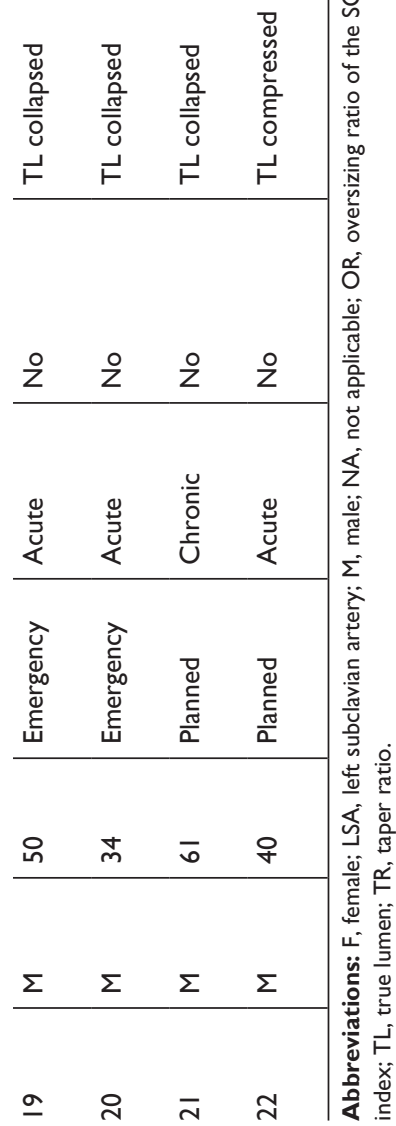

Table 3 Procedural details of the RBS technique

\begin{tabular}{l|l}
\hline Variable & Result $(\mathbf{n}=\mathbf{2 2})$ \\
\hline SG median length $(\mathrm{mm})$ & $181.2 \pm 23.9$ \\
Stent graft with taper & $7(31.8)$ \\
RBS & \\
$\quad$ Median diameter $(\mathrm{mm})$ & $24.7 \pm 2.7$ \\
$\quad$ Median length $(\mathrm{mm})$ & $126.3 \pm 66$ \\
$\quad$ Number of RBSs & $\mathrm{I} .6 \pm 0.8$ \\
Coverage of LSA & \\
$\quad$ Total coverage & $14(63.6)$ \\
Partial coverage & $5(22.7)$ \\
$\quad$ No coverage & $3(13.6)$ \\
Adjunctive procedures & \\
$\quad$ Chimney stent & 0 \\
Femoral-femoral bypass & $\mathrm{I}(4.5)$ \\
Mesenteric artery bare stent & $\mathrm{I}(4.5)$ \\
Mesenteric artery PTA & $\mathrm{I}(4.5)$ \\
Renal artery bare stent & 0 \\
\hline
\end{tabular}

Note: Continuous data are presented as mean \pm SD and categorical data are presented as number (\%).

Abbreviations: LSA, left subclavian artery; PTA, percutaneous angioplasty; RBS, restrictive bare stent; SG, stent graft.

diameter had also increased to $76.3 \mathrm{~mm}$. Considering the poor physical condition of the patient, we decided to follow a conservative policy with strict radiologic surveillance. At 18 months, the patient presented at the emergency department of a local hospital with hemodynamic collapse, and CTA revealed rupture of the chronic TBAD with aneurysm degeneration. Given the patient's extensive medical history, unfavorable prognosis, and poor condition, the decision was made to discontinue treatment.

Table 4 Outcomes and follow-up data

\begin{tabular}{l|l}
\hline Variable & Result $(\mathbf{n}=\mathbf{2 2})$ \\
\hline Days from onset to TEVAR & \\
Acute ( $\leq$ I4 days) & $8(36.4)$ \\
Subacute ( $>$ I4 days, $<3$ months) & $5(22.7)$ \\
Chronic ( $>3$ months) & $9(40.9)$ \\
TBAD with aneurysm degeneration & $6(31.8)$ \\
Complications & \\
Endoleak & 0 \\
Paraplegia & 0 \\
Stroke & 0 \\
Distal SINE & 0 \\
Acute renal failure & 0 \\
Bow and limb ischemia & 0 \\
RBS collapsed & $3(13.6)$ \\
Reintervention for all causes & $0(0)$ \\
30-day mortality & $0(0)$ \\
Overall aorta-related death & $1(4.5)$ \\
\hline
\end{tabular}

Note: Categorical data are presented as number (\%).

Abbreviations: RBS, restrictive bare stent; SINE, stent graft-induced new entry; TBAD, type B aortic dissection; TEVAR, thoracic endovascular aortic repair. 


\section{Discussion}

This study shows the flexibility and feasibility of the RBS technique, with a technical success rate of $100 \%$. It may not only improve descending aorta morphology but also promote TL expansion. No distal SINE, endoleak, or paraplegia occurred, and the 30-day mortality rate was $0 \%$. However, the rupture of chronic TBAD with aneurysm degeneration was not prevented in all patients. To our knowledge, this is the first study to comprehensively evaluate the descending aorta morphologic changes and to assess the outcomes of chronic TBAD with aneurysm after the RBS technique.

The TR and OR, which reflected local anatomy, are independent predictors of the development of SINE, and the cutoff values for predicting post-TEVAR SINE were 0.72 and 3.36, respectively. ${ }^{6,17,23}$ Our data showed that the post-RBS TR and OR values were significantly decreased compared with the preoperative values (TR $0.76 \pm 0.08-0.50 \pm 0.15$; OR 4.74 $\pm 2.68-1.93 \pm 1.03$ ), and no distal SINE was observed after the RBS procedure. Although preoperative use of tapered SGs $(7 / 22,31.8 \%)$ may also reduce the OR and TR, current tapered devices were designed with limited matches of the proximal and distal diameters. Thus, RBS is more flexible, as it can supply a better size match. Furthermore, our study also showed that this technique may help promote TL expansion and $95.4 \%$ of patients had a thrombosed FL in the segment of aortic coverage, which was higher than that in the 1-year follow-up results reported by Canaud et al. ${ }^{24}$

TI, which reflects the global anatomy, has been implicated in adverse outcomes after TEVAR. Chen et $\mathrm{al}^{25}$ associated high tortuosity with a higher rate of endoleak and strokes and with a lower survival rate. The spring-back force at the distal end of the SG across the tortuous descending aorta fixation site may make the dissected intima potentially vulnerable. However, the tortuous descending aorta was not involved in previous studies. ${ }^{17-20}$ In our experience, we should locate the distal landing zone in a relatively straight portion of the descending aorta. In our study, the results showed that an average of 1.6 RBSs were used to reduce the angle between the distal end of the stent and the aorta, the postoperative TI values decreased significantly $(1.44 \pm 0.17-1.32 \pm 0.16)$, and no distal SINE and endoleak were observed during follow-up. It is noteworthy that distal stent collapse is associated with severe tortuosity. Follow-up CTA revealed the collapse of RBSs in three patients with severe TI (TI $>6)$ and collapsed TL (patients 2, 3, and 4) in the absence of symptoms. The causes for these collapses are likely related to the inadequate radial forces of the distal stent. ${ }^{26}$
There are no reports of using the RBS technique for the repair of chronic TBAD with aneurysm degeneration. In our study, $40.9 \%$ were treated in the chronic phase, and $66.7 \%$ of the chronic TBAD cases presented as a chronic TBAD with aneurysm degeneration. One patient died from an aneurysm rupture. This may be interpreted from a pathological perspective. In our report, the percentages of patients with completely thrombosed FLs in the segments of the distal SG and the abdomen were unsatisfactory (63.6\% and $13.6 \%$, respectively). Thus, similar to PETTICOAT, the RBS technique failed to completely stop the blood from flowing backward to the thoracic FL. Moreover, in chronic dissection, the intimal membrane is thicker and more fibrotic than those in acute dissection, resulting in a reduced capacity for remodeling of the TL..$^{27,28}$ Therefore, instead of being related to distal stenting, the rupture may be the result of the dynamic evolution of aortic dissection pathology. As an adjunct one can use candy plugs or knickerbocker grafts, or simply occlude devices, to close the FL, and branched and fenestrated endovascular aortic repair can offer more opportunities in chronic TBAD with aneurysm degeneration. ${ }^{29}$

RBS selection was an important issue. The Sinus-XL stent is more accessible and affordable in People's Republic of China and is characterized by a closed-cell design with a large mesh size; it also has a higher radial force and can reduce the risk of spinal ischemia. Although distal bare stent systems including the Zenith Dissection Endovascular (Cook Medical) have been developed, the bare stent is designed for the PETTICOAT technique to provide intimal support, not restriction. Thus, we selected the Sinus-XL stent as the RBS. Furthermore, $10 \%$ oversizing of the SG is recommended, but the descending aorta of a TBAD is always dissected so $10 \%$ oversizing of a RBS may injure the intimal flap. In our report, we selected size of the RBS on the basis of the long diameter of the TL at the intended distal edge of the RBS, and no oversizing was adopted. During follow-up, the mean diameter of the RBS $(24.7 \pm 2.7 \mathrm{~mm})$ was consistent with the results of previous reports ${ }^{17-19}$ and no dislodging or disjointing of the RBS was observed.

The primary limitations of this study include the small number of patients, which may account for the underestimation of technical concerns or possible complications associated with the RBS. A comparison of patients treated with RBS with patients anatomically suitable for but not receiving RBS would be helpful to establish the true value of this technique, but this comparison was not possible in this retrospective review. In addition, a longer follow-up period is needed to validate the results and to draw any 
definitive conclusions about the safety and durability of the RBS technique.

\section{Conclusion}

The RBS technique may effectively improve descending aorta morphologic remodeling, potentially addressing the limitations of current grafts. However, the rupture of chronic TBAD with aneurysm degeneration was not prevented by the RBS technique in all patients. Further prospective clinical studies are warranted to evaluate the procedure's long-term efficacy.

\section{Disclosure}

The author reports no conflicts of interest in this work.

\section{References}

1. Tsai TT, Fattori R, Trimarchi S, et al. Long-term survival in patients presenting with type $\mathrm{B}$ acute aortic dissection: insights from the International Registry of Acute Aortic Dissection. Circulation. 2006;114(21): 2226-2231.

2. Eriksson MO, Steuer J, Wanhainen A, et al. Morphologic outcome after endovascular treatment of complicated type B aortic dissection. $J$ Vasc Interv Radiol. 2013;24(12):1826-1833.

3. Dong Z, Fu W, Wang Y, et al. Stent graft-induced new entry after endovascular repair for Stanford type B aortic dissection. J Vasc Surg. 2010;52(6):1450-1457.

4. Thrumurthy SG, Karthikesalingam A, Patterson BO, et al. A systematic review of mid-term outcomes of thoracic endovascular repair (TEVAR) of chronic type B aortic dissection. Eur J Vasc Endovasc Surg. 2011;42(5):632-647.

5. Neuhauser B, Greiner A, Jaschke W, Chemelli A, Fraedrich G. Serious complications following endovascular thoracic aortic stent-graft repair for type B dissection. Eur J Cardiothorac Surg. 2008;33(1):58-63.

6. Weng SH, Weng CF, Chen WY, et al. Reintervention for distal stent graft-induced new entry after endovascular repair with a stainless steelbased device in aortic dissection. J Vasc Surg. 2013;57(1):64-71.

7. Chen IM, Huang CY, Weng SH, et al. Implantation sequence modification averts distal stent graft-induced new entry after endovascular repair of Stanford type B aortic dissection. J Vasc Surg. 2016;64(2): 281-288.

8. Jánosi RA, Tsagakis K, Bettin M, et al. Thoracic aortic aneurysm expansion due to late distal stent graft-induced new entry. Catheter Cardiovasc Interv. 2015;85(2):E43-E53.

9. Pantaleo A, Jafrancesco G, Buia F, et al. Distal Stent Graft-Induced New Entry: An Emerging Complication of Endovascular Treatment in Aortic Dissection. Ann Thorac Surg. 2016;102(2):527-532.

10. Li Q, Wang LF, Ma WG, et al. Risk factors for distal stent graft-induced new entry following endovascular repair of type B aortic dissection. J Thorac Dis. 2015;7(11):1907-1916.

11. Huang CY, Weng SH, Weng CF, et al. Factors predictive of distal stent graft-induced new entry after hybrid arch elephant trunk repair with stainless steel-based device in aortic dissection. J Thorac Cardiovasc Surg. 2013;146(3):623-630.
12. Resch TA, Delle M, Falkenberg M, et al. Remodeling of the thoracic aorta after stent grafting of type B dissection: a Swedish multicenter study. J Cardiovasc Surg. 2006;47(5):503-508.

13. Xu SD, Huang FJ, Du JH, et al. A study of aortic dimension in type B aortic dissection. Interact Cardiovasc Thorac Surg. 2008;7(2):244-248.

14. Senf B, von Sachsen S, Neugebauer R, et al. The effect of stent graft oversizing on radial forces considering nitinol wire behavior and vessel characteristics. Med Eng Phys. 2014;36(11):1480-1486.

15. Eggebrecht $\mathrm{H}$, Nienaber $\mathrm{CA}$, Neuhäuser $\mathrm{M}$, et al. Endovascular stentgraft placement in aortic dissection: a meta-analysis. Eur Heart $J$. 2006;27(4):489-498.

16. Nienaber CA, Kische S, Zeller T, et al. Provisional extension to induce complete attachment after stent-graft placement in type B aortic dissection: the PETTICOAT concept. $J$ Endovasc Ther. 2006;13(6): 738-746.

17. Zhao Y, Yin H, Chen Y, et al. Restrictive bare stent prevents distal stent graft-induced new entry in endovascular repair of type B aortic dissection. J Vasc Surg. 2018;67(1):93-103.

18. Feng J, Lu Q, Zhao Z, et al. Restrictive bare stent for prevention of stent graft-induced distal redissection after thoracic endovascular aortic repair for type B aortic dissection. J Vasc Surg. 2013;57(2 Suppl):44S-52S.

19. He H, Yao K, Nie WP, et al. Modified Petticoat Technique with Pre-placement of a Distal Bare Stent Improves Early Aortic Remodeling after Complicated Acute Stanford Type B Aortic Dissection. Eur J Vasc Endovasc Surg. 2015;50(4):450-459.

20. Liu JF, Jiang WL, Lu HT, Li YL, Zhang TH, Yamakawa T. Application of protective stents in endovascular repair of acute complicated Stanford type B aortic dissections. J Endovasc Ther. 2013;20(2):210-218.

21. Shi Z, Yang J, Fu W, et al. Outcomes and aortic remodelling after proximal thoracic endovascular aortic repair of post type B aortic dissection thoracic aneurysm. Vasa. 2016;45(4):331-336.

22. Shirali AS, Bischoff MS, Lin HM, et al. Predicting the risk for acute type B aortic dissection in hypertensive patients using anatomic variables. JACC Cardiovasc Imaging. 2013;6(3):349-357.

23. Jang H, Kim MD, Kim GM, et al. Risk factors for stent graft-induced new entry after thoracic endovascular aortic repair for Stanford type B aortic dissection. J Vasc Surg. 2017;65(3):676-685.

24. Canaud L, Patterson BO, Peach G, et al. Systematic review of outcomes of combined proximal stent grafting with distal bare stenting for management of aortic dissection. J Thorac Cardiovasc Surg. 2013;145(6): 1431-1438.

25. Chen CK, Liang IP, Chang HT, et al. Impact on outcomes by measuring tortuosity with reporting standards for thoracic endovascular aortic repair. J Vasc Surg. 2014;60(4):937-944.

26. Massmann A, Kunihara T, Fries P, et al. Uncovered stent implantation in complicated acute aortic dissection type B. J Thorac Cardiovasc Surg. 2014;148(6):3003-3011.

27. Peterss S, Mansour AM, Ross JA, et al. Changing Pathology of the Thoracic Aorta From Acute to Chronic Dissection: Literature Review and Insights. J Am Coll Cardiol. 2016;68(10):1054-1065.

28. Fanelli F, Cannavale A, O'Sullivan GJ, et al. Endovascular Repair of Acute and Chronic Aortic Type B Dissections: Main Factors Affecting Aortic Remodeling and Clinical Outcome. JACC Cardiovasc Interv. 2016;9(2):183-191.

29. Schepens M. Type B aortic dissection: new perspectives. J Vis Surg. 2018;4:75-83 
Therapeutics and Clinical Risk Management

Dovepress

\section{Publish your work in this journal}

Therapeutics and Clinical Risk Management is an international, peerreviewed journal of clinical therapeutics and risk management, focusing on concise rapid reporting of clinical studies in all therapeutic areas, outcomes, safety, and programs for the effective, safe, and sustained use of medicines. This journal is indexed on PubMed Central, CAS,

EMBase, Scopus and the Elsevier Bibliographic databases. The manuscript management system is completely online and includes a very quick and fair peer-review system, which is all easy to use. Visit http://www.dovepress.com/testimonials.php to read real quotes from published authors.

Submit your manuscript here: http://www.dovepress.com/therapeutics-and-clinical-risk-management-journal 